\title{
Distances and ages of NGC 6397, NGC 6752 and 47 Tuc $^{\star}$
}

\author{
R. G. Gratton ${ }^{1}$, A. Bragaglia ${ }^{2}$, E. Carretta ${ }^{1}$, G. Clementini $^{2}$, S. Desidera ${ }^{1}$, F. Grundahl ${ }^{3}$, and S. Lucatello ${ }^{1,4}$ \\ 1 INAF-Osservatorio Astronomico di Padova, Vicolo dell'Osservatorio 5, 35122 Padova, Italy \\ 2 INAF-Osservatorio Astronomico di Bologna, Via Ranzani 1, 40127 Bologna, Italy \\ 3 Institute of Physics and Astronomy, Aarhus University, Ny Munkegade, 8000 Aarhus C, Denmark \\ ${ }^{4}$ Dipartimento di Astronomia, Università di Padova, Italy, Vicolo dell'Osservatorio 2, 35122 Padova, Italy
}

Received 28 April 2003 / Accepted 23 June 2003

\begin{abstract}
New improved distances and absolute ages for the Galactic globular clusters NGC 6397, NGC 6752, and 47 Tuc are obtained using the Main Sequence Fitting Method. We derived accurate estimates of reddening and metal abundance for these three clusters using a strictly differential procedure, where the Johnson $B-V$ and Strömgren $b-y$ colours and UVES high resolution spectra of turn-off stars and early subgiants belonging to the clusters were compared to similar data for field subdwarfs with accurate parallaxes measured by Hipparcos. The use of a reddening free temperature indicator (the profile of $\mathrm{H} \alpha$ ) allowed us to reduce the error bars in reddening determinations to about $0.005 \mathrm{mag}$, and in metal abundances to 0.04 dex, in the scales defined by the local subdwarfs. Error bars in distances are then reduced to about 0.07 mag for each cluster, yielding ages with typical random errors of about 1 Gyr. We find that NGC 6397 and NGC 6752 have ages of $13.9 \pm 1.1$ and $13.8 \pm 1.1 \mathrm{Gyr}$ respectively, when standard isochrones without microscopic diffusion are used, while 47 Tuc is probably about 2.6 Gyr younger, in agreement with results obtained by other techniques sensitive to relative ages. If we use models that include the effects of sedimentation due to microscopic diffusion in agreement with our observations of NGC 6397, and take into account various sources of possible systematic errors with a statistical approach, we conclude that the age of the oldest globular clusters in the Galaxy is $13.4 \pm 0.8 \pm 0.6 \mathrm{Gyr}$, where the first error bar accounts for random effects, and the second one for systematic errors. This age estimate is fully compatible with the very recent results from WMAP, and indicates that the oldest Galactic globular clusters formed within the first $1.7 \mathrm{Gyr}$ after the Big Bang, corresponding to a redshift of $z \geq 2.5$, in a standard $\Lambda \mathrm{CDM}$ model. The epoch of formation of the (inner halo) globular clusters lasted about $2.6 \mathrm{Gyr}$, ending at a time corresponding to a redshift of $z \geq 1.3$. On the other hand, our new age estimate once combined with values of $H_{0}$ given by WMAP and by the HST Key Project, provides a robust upper limit at 95\% level of confidence of $\Omega_{M}<0.57$, independently of type Ia SNe, and strongly supports the need for a dark energy. The new cluster distances lead to new estimates of the horizontal branch luminosity, that may be used to derive the zero point of the relation between the horizontal branch absolute magnitude and metallicity: we obtain $M_{V}(H B)=(0.22 \pm 0.05)([\mathrm{Fe} / \mathrm{H}]+1.5)+(0.56 \pm 0.07)$. This zero point is 0.03 mag shorter than obtained by Carretta et al. (2000) and within the error bar it agrees with, but it is more precise than most of the previous individual determinations of the RR Lyrae absolute magnitude. When combined with the apparent average luminosity of the RR Lyrae stars in the LMC by Clementini et al. (2003), this zero point provides a new estimate of the distance modulus to the LMC: $(m-M)_{0}=18.50 \pm 0.09$.
\end{abstract}

Key words. stars: abundances - stars: evolution - stars: Population II - Galaxy: globular clusters: general - Galaxy: formation - cosmology: distance scale

\section{Introduction}

Globular clusters are among the oldest objects in our Galaxy. Their ages provide basic informations on the early stages of Galactic formation, and give lower limits to the age of the Universe. This latter issue has become in the last years less urgent, since the increasing evidences for an acceleration in the Universe expansion have lead to older ages for the Universe. However, when coupled with determinations of the Hubble

Send offprint requests to: R. G. Gratton,

e-mail: gratton@pd.astro.it

* Based on data collected at the European Southern Observatory, Chile, telescopes (program 165.L-0263). constant $H_{0}$ from the WMAP experiment (Spergel et al. 2003) and from the HST Key Project (Freedman et al. 2001), accurate ages of globular clusters can be used to constrain the value of $\Omega_{\mathrm{M}}$ independently of observations of type Ia $\mathrm{SNe}$ (Perlmutter et al. 1999). This is an important issue given the existing concerns about systematic effects on the maximum brightness of type Ia SNe occurring at high redshift (see e.g. Dominguez et al. 2001). Once the presence of dark energy is assumed, globular cluster ages can be used to constrain a time average of the exponent of the equation of state of the vacuum energy $w$ (Jimenez et al. 2003), possibly allowing to discriminate between various models (strings, vacuum energy, quintessence: see Wang et al. 2000). On the other side, assuming a standard 
$\Lambda \mathrm{CDM}$ model, accurate ages for globular clusters and for the Universe (within this framework fixed at $13.7 \pm 0.2 \mathrm{Gyr}$ by the WMAP measurements: Bennett et al. 2003) allow to compare the epoch of formation of our Galaxy with the evolution of high redshift galaxies, and to put it into a cosmological framework (see Carretta et al. 2000; Freeman \& Bland-Hawthorn 2002).

Ages require clocks; in the case of globular clusters, three major dating techniques may be considered. First, nucleocosmochronology provides ages by comparing abundances of unstable isotopes with those of stable ones. Widely used in geology, this technique is much more difficult to apply in astronomy because we may only derive accurate abundances for a few elements, that are often not tied to each other by simple decaying mechanisms. Assumptions about nucleosynthesis are needed. Long living unstable elements like Th and more recently $\mathrm{U}$ have been used to derive ages in a few old, very metal-poor stars by comparing their abundances with those of other stable nuclei synthesized by the $r$-process (see Sneden et al. 2001; Cayrel et al. 2001; Cowan et al. 2002). Recently Hill et al. (2002) obtained an age of $14.0 \pm 2.4 \mathrm{Gyr}$ for the extremely metal-poor star CS 31082-001, employing this technique. In spite of these admirable efforts, real uncertainties of such determinations are difficult to assess and are likely large, because they depend on the adopted model for the $r$-process that is still quite uncertain (see e.g. Truran et al. 2002).

A second clock relies on the final part of the cooling sequence for white dwarfs, where it is possible to exploit the well defined maximum of the luminosity function due to the formation of $\mathrm{H}_{2}$ molecules in the atmospheres (Richer et al. 2000). Recently, a spectacular, extremely deep colour magnitude for M 4 obtained using HST has allowed Hansen et al. (2002) to derive an age of $12.7 \pm 0.7$ Gyr for this cluster. Again the error bar is uncertain, and De Marchi et al. (2002) showed that the maximum of the luminosity function has not yet been reached, and that the adoption of different sets of evolutionary models for white dwarfs would lead to quite different ages. In fact the only solid conclusion that can be reached at present with this method is that the age of globular clusters is larger than $10 \mathrm{Gyr}$.

The third clock uses the luminosity of the turn-off (TO) from the main sequence: this is a well established method, whose uncertainties have long been studied in detail (see Renzini \& Fusi Pecci 1988). Model uncertainties have been carefully considered by several authors (see e.g. Chaboyer et al. 1996); the main source of remaining uncertainty is related to the treatment of microscopic diffusion and levitation due to radiation pressure. These mechanisms are required to produce solar models that agree with evidences from helioseismology. Models which include microscopic diffusion (using a complete ionization approach) lead to ages for globular clusters that are systematically smaller by about 1 Gyr than those predicted by models where diffusion is neglected. Other sources of uncertainties (treatment of subatmospheric convection, details of the used code, and of the normalization to the Solar values) have a smaller impact (typically less than $0.5 \mathrm{Gyr}$ ). Chaboyer et al. (1996) showed how all these uncertainties can be treated in a statistical way, leading to a reliable prediction for the error bar.
However, the main source of errors in ages from the TO luminosity actually comes from errors in the distances (that also affect ages estimated from the end of the white dwarf cooling sequence, but notably not those from nucleocosmochronology). Typically, an error of 0.07 mag in distance moduli (that is, a mere $3.5 \%$ in distances) leads to an error of about $1 \mathrm{Gyr}$ in ages. Due to the large sensitivity of ages on distances, several investigators have considered dating techniques that are independent of distance ${ }^{1}$; however, most of them only provide relative ages to the clusters that are still very useful in studies of Galactic formation and evolution. Relative ages for globular clusters accurate to better than $1 \mathrm{Gyr}$ can be derived mainly with two techniques: the difference in magnitude between the horizontal branch (HB) at the average colour of the RR Lyrae stars and the TO (the so-called vertical method), and the difference in colours between the TO and the base of the red giant branch (RGB) (the so-called horizontal method). Rosenberg et al. (1999) showed that the relative rankings obtained with these two techniques agree well. We will use Rosenberg et al. results in our discussion, that is however focused on absolute ages.

Derivation of distances to globular clusters at the accuracy needed for their dating is a difficult task. In perspective, important progresses are expected in the next few years from the dynamical methods that compare proper motions obtained using HST with extensive sets of radial velocities obtained from new generation fiber fed spectrographs like FLAMES, by means of suitable dynamical models for the clusters (see King \& Anderson 2002; Bedin et al. 2003). Relevant data will be obtained in the next years, and there is sound hope to derive robust distance estimates accurate to a few per cent level for several clusters. In the meantime, we may try to improve other techniques to provide a comparable accuracy for at least some clusters. In this paper new distances for three of the four clusters closest to the Sun are derived using the Main Sequence Fitting Method and data acquired within the ESO Large Program 165.L-0263, at the VLT telescope. In Sect. 2 we discuss the most critical issues within this method, and our strategy to reduce the $1-\sigma$ error bar from the previous value of $\pm 0.12 \mathrm{mag}$ (Carretta et al. 2000) to \pm 0.07 mag. In Sect. 3 we present the observational data (both photometry and high resolution spectroscopy) on which our new analysis is based. In Sect. 4 we present our new derivation of reddening, metallicity, and distance to the clusters. In Sect. 5 we use our new values of the distances to these three clusters to estimate the zero point of the luminosity-metallicity relation for RR Lyrae stars and discuss the impact of our results on the distance to the Large Magellanic Cloud (LMC). Finally, in Sect. 6 we obtain ages for the clusters, compare them with previous estimates, and briefly

\footnotetext{
${ }^{1}$ For instance, Chaboyer \& Krauss (2002) have recently derived the age of the double-lined detached binary OGLE17GC in $\omega$ Cen from the location of the two components in the colour-magnitude diagram and their masses, independently of the cluster distance. The derived age $t=11.1 \pm 0.7 \mathrm{Gyr}$ is compatible with those obtained for the clusters analyzed in the present paper. We also note that $\omega$ Cen is a very peculiar object, perhaps the nucleus of a now dissolved nucleated dwarf elliptical, and its age may well be different from the age of the bulk of Galactic globular clusters.
} 
Table 1. Error budget of distance moduli based on the Main Sequence Fitting Method before and after this paper.

\begin{tabular}{ccc}
\hline \hline Effect/involved error & $\Delta(m-M)$ & $\Delta(m-M)$ \\
& Before & After \\
\hline
\end{tabular}

Local sample properties

$\begin{array}{lcc}\text { Parallax error } & \pm 0.01 & \pm 0.01 \\ \text { Malmquist bias } & \text { negligible } & \text { negligible } \\ \text { Lutz-Kelker correction } & \pm 0.02 & \pm 0.02\end{array}$

Binary contamination

$\begin{array}{lll}\text { Field binaries } & \pm 0.02 & \pm 0.02 \\ \text { Cluster binaries } & \pm 0.02 & \pm 0.02\end{array}$

Systematic differences

\begin{tabular}{lcc} 
Phot. calibrations $(0.01 \mathrm{mag})$ & \pm 0.04 & \pm 0.04 \\
Reddening scale $(0.015 \mathrm{mag})$ & \pm 0.07 & \pm 0.035 \\
Metallicity scale $(0.1 \mathrm{dex})$ & \pm 0.08 & $\left(^{*}\right)$ \\
& & \\
Total Uncertainty & \pm 0.12 & \pm 0.07 \\
\hline
\end{tabular}

(*) The effect of an error in the metallicity were included into the error bar due to reddening (see text).

discuss their impact on the age of the Universe and the epoch of formation of the Milky Way (MW).

\section{The Main Sequence Fitting method: Critical issues}

Prompted by the release of the high precision parallaxes measured by the ESA Hipparcos satellite (Perryman et al. 1997), numerous determinations of distances to several Galactic globular clusters using the Main Sequence Fitting (MSF) method appeared in the the literature in recent years (Reid 1997, 1998; Gratton et al. 1997; Pont et al. 1998; Carretta et al. 2000; Percival et al. 2002; Grundahl et al. 2002). As a general result all these studies produced cluster distances distinctly longer by about $15 \%$ (that is $0.3 \mathrm{mag}$ in the distance moduli) than those obtained previously using the same technique, although with some differences from one author to the other. As outlined in Gratton et al. (1997) this result is simply because distances to the local subdwarfs obtained with the Hipparcos parallaxes are larger than those obtained with the much more uncertain ground based parallaxes.

While the Main Sequence Fitting Method (MSF) is probably the best understood among the techniques used to derive distances to globular clusters, there are a number of subtleties that should be carefully considered when accuracies to a few per cent level are required. In depth discussions of the most relevant sources of errors were presented in Gratton et al. (1997), Pont et al. (1998), and Carretta et al. (2000). These last authors published a table listing the major contributors to the total error budget affecting the MSF distances, that we have reproduced in the first two columns of Table 1. Errors may be divided into three separate groups: the first group is more directly related to the properties of the local subdwarf sample, and includes errors in individual parallaxes, Malmquist bias, and the Lutz-Kelker correction. The main contribution by Hipparcos was to reduce these error sources to very small values, at least for stars and clusters with metallicities larger than $[\mathrm{Fe} / \mathrm{H}]=-2$. The second group concerns possible contamination by binaries both of field stars and globular cluster mean loci. Pont et al. (1998) suggested a rather large binary correction, however their result was criticized by Gratton et al. (1997) and Carretta et al. (2000), who concluded that once known or suspected binaries are eliminated from the sample, the residual correction for still undetected binaries is likely to be within a few hundredths of a magnitude.

Finally, the last group of errors listed in Table 1 concerns possible systematic differences between field and cluster stars in various parameters adopted in the analysis. They generally affect the colours of the main sequence, and their impact on the distance determinations is due to the rather steep slope of the main sequence (roughly, $\Delta M_{V} \sim 5 \Delta(B-V)$ ) in the colour magnitude diagram. The MSF is influenced by systematic errors (given in parenthesis in Table 1) in the photometric calibrations, in the adopted values for the interstellar reddening, and in the metallicity scales. According to Table 1, these are by far the largest contributors to the total error bar. Could they possibly cancel out, errors in the MSF distance moduli would be reduced to $0.04 \mathrm{mag}$, that is an error of only $2 \%$ in distance, and of $0.6 \mathrm{Gyr}$ in age. While the first two terms in this group (namely photometric calibration and reddening) directly affect the colours of the stars, the third one (metallicity) acts indirectly, through the dependence on metallicity of the main sequence colour in the commonly used bands (mainly $B-V$ ). Systematic differences in reddenings may arise from uncertainties in the scale height of interstellar dust, since local subdwarfs are within the dust absorbing layer, while globular clusters are much farther. At the same time, systematic differences in metallicities may be present when comparing abundances obtained from analysis of local subdwarfs and giants in globular clusters.

In order to significantly reduce the error in the cluster distances derived with the MSF method, we need then to deal with all these various sources of errors. We note that all of them could be substantially reduced if we were able to obtain temperatures and metal abundances for both field and cluster main sequence stars using the same colour-independent technique, thus by-passing uncertainties related to colours. This was, in fact, our approach; however, since the present instrumentation still does not allow to derive temperatures and colours directly for faint unevolved main sequence stars in globular clusters, we used instead slightly brighter stars near the TO and at the base of the RGB. For these stars temperatures were derived by fitting the profile of $\mathrm{H} \alpha$ in high resolution spectra obtained with UVES at the Kueyen telescope (ESO VLT Unit 2). Comparisons of the colour-temperature relations for field (assumed to be unreddened) and cluster stars allowed us to derive the reddening of the clusters. Actually, these reddening values depend on the adopted photometries, so that a zero point error in the photometric calibration directly translates into a corresponding error in the reddening. However, apparent 
Table 2. Unevolved bona fide single local subdwarfs.

\begin{tabular}{|c|c|c|c|c|c|c|c|c|}
\hline $\mathrm{HD} / \mathrm{DM}$ & $\begin{array}{c}\pi \\
\text { mas }\end{array}$ & $V$ & $M_{V}$ & $B-V$ & $b-y$ & {$[\mathrm{Fe} / \mathrm{H}]$} & {$[\alpha / \mathrm{Fe}]$} & {$[\mathrm{M} / \mathrm{H}]$} \\
\hline \multicolumn{9}{|c|}{ Program stars } \\
\hline-350360 & $16.28 \pm 1.76$ & 10.25 & $6.31 \pm 0.23$ & 0.765 & 0.469 & -1.15 & 0.39 & -0.86 \\
\hline 25329 & $54.14 \pm 1.08$ & 8.51 & $7.18 \pm 0.04$ & 0.870 & 0.529 & -1.80 & 0.49 & -1.43 \\
\hline 75530 & $18.78 \pm 1.48$ & 9.19 & $5.56 \pm 0.17$ & 0.734 & 0.445 & -0.61 & 0.31 & -0.39 \\
\hline-800328 & $16.46 \pm 0.99$ & 10.10 & $6.18 \pm 0.13$ & 0.576 & 0.423 & -2.03 & 0.17 & -1.91 \\
\hline 103095 & $109.22 \pm 0.78$ & 6.45 & $6.64 \pm 0.02$ & 0.750 & 0.484 & -1.33 & 0.29 & -1.12 \\
\hline 120559 & $40.02 \pm 1.00$ & 7.97 & $5.98 \pm 0.05$ & 0.664 & 0.424 & -0.94 & 0.33 & -0.70 \\
\hline 126681 & $19.16 \pm 1.44$ & 9.32 & $5.73 \pm 0.16$ & 0.597 & 0.400 & -1.14 & 0.32 & -0.91 \\
\hline 134439 & $34.14 \pm 1.36$ & 9.07 & $6.74 \pm 0.09$ & 0.777 & 0.486 & -1.38 & 0.12 & -1.30 \\
\hline 134440 & $33.68 \pm 1.67$ & 9.44 & $7.08 \pm 0.11$ & 0.854 & 0.522 & -1.45 & 0.19 & -1.32 \\
\hline 145417 & $72.75 \pm 0.82$ & 7.52 & $6.83 \pm 0.02$ & 0.820 & 0.505 & -1.39 & 0.34 & -1.15 \\
\hline+224454 & $17.66 \pm 1.44$ & 9.50 & $5.73 \pm 0.18$ & 0.770 & 0.459 & -0.60 & 0.30 & -0.39 \\
\hline
\end{tabular}

\section{Additional stars}

\begin{tabular}{lllllllll}
104006 & $31.35 \pm 1.05$ & 8.89 & $6.38 \pm 0.07$ & 0.816 & 0.492 & -0.81 & $(0.29)$ & -0.60 \\
108564 & $35.30 \pm 1.20$ & 9.43 & $7.16 \pm 0.07$ & 0.976 & 0.563 & -0.72 & $(0.28)$ & -0.52 \\
123505 & $20.95 \pm 1.65$ & 9.67 & $6.27 \pm 0.17$ & 0.782 & 0.475 & -0.72 & $(0.28)$ & -0.52 \\
145598 & $26.04 \pm 1.34$ & 8.65 & $5.73 \pm 0.11$ & 0.662 & 0.425 & -0.79 & $(0.28)$ & -0.59 \\
\hline
\end{tabular}

distances (that is, not corrected for the interstellar absorption) are unaffected by these errors. The same high resolution spectra were used to perform an abundance analysis both for field and cluster stars using strictly the same procedure. In this way, we were able to obtain reddening and metallicity scales for globular clusters with an accuracy only limited by the dispersion of individual temperature derivations. It is unfortunate that this is still not negligible so that the error due to the reddening scale, while reduced to about half of the original value given in Table 1, is still the largest individual source of error. Also, a significant contribution to the error bar is likely due to residual, colour dependent photometric errors, that do not cancel out using our procedure. Nevertheless, our distance derivations, with errors of about $3.5 \%$, are the most accurate presently available for globular clusters, and allow a substantial progress in the absolute age derivation.

\section{Observational data}

The observational data used in this paper were obtained within the ESO Large Program 165.L-0263. In the present analysis we consider only three of the globular clusters observed within that project, namely NGC 6397, NGC 6752, and 47 Tuc. These clusters were selected because they are the closest to the Sun, with the only exception of NGC 6121 (M 4), that is known to be affected by differential interstellar reddening which greatly complicates its analysis (see e.g. Ivans et al. 1999). For each of the selected clusters we obtained high resolution spectra at a resolution of about 40000 and $20<S / N<100$ for stars near the TO, and at the base of the RGB. With the same set up we also acquired spectra of about thirty local subdwarfs selected from the sample in Carretta et al. (2000). All of them have parallaxes measured by Hipparcos with errors $\Delta \pi / \pi<0.12$. Informations about binarity are available for most of these stars: they were used to select an appropriate sample of bona fide single stars adopted in the distance derivations. Informations on the unevolved $\left(M_{V}>5.5\right)$ bona fide single local subdwarf are given in the first part of Table 2.

Details of data aquisition and abundance analysis have been given elsewhere (Gratton et al. 2001, 2003; Carretta et al. 2003), and here we will recall only a few relevant points.

\subsection{Photometry}

While an enormous amount of high quality photometric data has been obtained in the last years for globular clusters, most of it could not be used in the present analysis since these photometries are mainly in the $R$ and $I$ photometric bands and we lack comparable data for the local subdwarfs. The same applies to the excellent HST data. We preferred to avoid uncertain colour transformations (see Clementini et al. 1999 for a discussion), and throughout this paper we then used only 
two sets of photometric data existing both for field and cluster stars, namely the Johnson $B V$ and the Strömgren $u v b y$ photometry. Johnson $B V$ photometry for the program clusters was taken from Alcaino et al. (1997) and Kaluzny (1997) for NGC 6397; from Thompson et al. (1999) for NGC 6752; and from Hesser et al. (1987), corrected according to the prescriptions in Percival et al. (2002), for 47 Tuc. Strömgren $u v b y$ photometry was obtained by Grundahl \& Andersen (1999). We will assume hereinafter that all these photometries are in the standard systems.

Johnson and Strömgren photometric data for the field stars were obtained from the Simbad database: for the $B V$ photometry we simply averaged the various entries given there, while for the Strömgren photometry we adopted the values recommended by Hauck \& Mermilliod (1998). The adopted photometric data for the field stars are given in Gratton et al. (2003) and also provided in Table 2 for the stars used in the distance derivations. Reddening for the field subdwarfs is discussed by Schuster \& Nissen (1989) and Carney et al. (1994), and generally found to be small or zero. Hereinafter we will assume that the field stars are unreddened.

The absolute magnitudes listed in Table 2 do not include the Lutz-Kelker (1973) correction. This is uncertain, but it is small for our sample (the maximum value for an individual star being $\sim 0.07 \mathrm{mag}$ ). In our distance derivations, we have corrected for the Lutz-Kelker effect using Eq. (1) of Gratton et al. (1997), that probably slightly overestimates this correction. However, cluster distance moduli would only be 0.01 mag longer we neglect if this correction.

\subsection{High resolution spectroscopy}

The high resolution spectra were used to derive effective temperatures from the profile of $\mathrm{H} \alpha$, and the chemical composition from the analysis of weak metal lines. These analyses are described in Gratton et al. (2001), Carretta et al. (2003), and Gratton et al. (2003). The same procedure was adopted both for field and cluster stars.

For the analysis of the $\mathrm{H} \alpha$ line we compared the observed spectral profiles with those derived using Kurucz (1994) models with the overshooting option switched off. Synthetic profiles were computed with the same precepts adopted by Castelli et al. (1997). An example of fit obtained with this technique is given in Gratton et al. (2003); note that appropriate gravities and metallicities were adopted when synthesizing line profiles. Temperatures derived with this approach have typical errors of about $150 \mathrm{~K}$. This rather large uncertainty is due to difficulties related to a proper flat fielding of the echelle spectra provided by the $31.6 \mathrm{gr} / \mathrm{mm}$ grating that has a rather limited free spectral range. Figure 6 of Gratton et al. (2003) compares the effective temperatures obtained by this technique with those obtained from colours for the field stars. The rms of the relation is $159 \mathrm{~K}$. Similar uncertainties are derived from the star-to-star scatter for stars in clusters. Ten to eighteen stars were considered in each cluster; errors in the zero points of the temperatures are then from 35 to $50 \mathrm{~K}$ for individual clusters, to be added quadratically to a similar uncertainty for the field stars (about $27 \mathrm{~K}$ ).

\section{Reddening, metallicity and distances}

\subsection{Reddening}

Estimates of the interstellar reddening toward the selected clusters were obtained by comparing the observed colourtemperature relations for the field subdwarfs with that determined for the globular cluster stars. When stars of similar metallicity and gravity are considered, the reddening is simply given by the offset between the two relations. However, the field stars generally have both metallicities and gravities slightly different from those of the cluster stars, so we inverted the colour-temperature relations given by Kurucz (1994) model atmospheres both for field and globular cluster stars, using surface gravities given by the location of the stars in the colour-magnitude diagram and masses from fittings to $14 \mathrm{Gyr}$ old isochrones (Girardi et al. 2002), and metallicities from our abundance analysis (see Sect. 4.2). We then compared these colours with those observed for the field and globular cluster stars: the offsets between colours are our reddening estimates.

As noticed by the referee, reddenings obtained by this procedure are sensitive to the selected colour transformations. Somewhat different results would be obtained by replacing Kurucz transformations with those e.g. from Houdashelt et al. (2000; we preferred the former ones because they consider both $B-V$ and $b-y$ colours). Replacing Kurucz transformations with those from Houdashelt et al., and repeating the whole procedure, we find that the reddening from $B-V$ reduces by as much as 0.02 mag for NGC 6397, while changes are about half this value for NGC 6752, and very small for 47 Tuc. We expect that uncertainties in the transformations are much less important for the intermediate band $b-y$ colour than for the wide band $B-V$ one. However, this cannot be verified since Houdashelt et al. (2000) do not provide predictions for $b-y$.

Three things must be noticed here: (i) a strong support to the transformations we have adopted is provided by the very good agreement between the temperature scale adopted in our analysis and that obtained using the Infrared Flux Method by Alonso et al. (1996; see Gratton et al. 2003: note that this excellent agreement is obtained for the average of temperatures from $B-V$ and $b-y$ colours). (ii) Both cluster subgiants and TO-stars were used, and results averaged: typically, TO-stars have temperatures of about 5800-6300 K, and subgiants of about 5100-5500 K, depending on the cluster. (iii) The field stars used in the cluster reddening derivation are those listed in Gratton et al. (2003) having temperatures from $\mathrm{H} \alpha$. A total of 35 stars were used; they have $4900<T_{\text {eff }}<6300 \mathrm{~K}$, $3.4<\log g<4.8$, and $-2.2<[\mathrm{M} / \mathrm{H}]<-0.4$, so that they effectively overlap with the cluster stars, reducing the impact of offsets due to uncertainties in the temperature-colour transformations used as intermediate steps in our reddening derivations. This is confirmed by the good agreement between the reddening values obtained from subgiants and TO-stars: on average, the difference for the tree clusters is $0.006 \pm 0.005 \mathrm{mag}$ (reddenings from TO-stars being slightly smaller), and in no case the difference between the values obtained from TO-stars and subgiants is larger than the $1 \sigma$ internal error bar. 
Table 3. Reddening estimates for the three globular clusters.

\begin{tabular}{lccc}
\hline \hline Source & $\begin{array}{c}E(B-V) \\
\text { NGC 6397 }\end{array}$ & $\begin{array}{c}E(B-V) \\
\text { NGC 6752 }\end{array}$ & $\begin{array}{c}E(B-V) \\
47 \text { Tuc }\end{array}$ \\
\hline Stars used & 10 & 18 & 12 \\
$b-y$ & $0.178 \pm 0.007$ & $0.045 \pm 0.007$ & $0.021 \pm 0.005$ \\
$B-V$ & $0.186 \pm 0.006$ & $0.035 \pm 0.007$ & $0.035 \pm 0.009$ \\
average & $0.183 \pm 0.005$ & $0.040 \pm 0.005$ & $0.024 \pm 0.004$ \\
Harris (1996) & 0.18 & 0.04 & 0.05 \\
Schlegel et al. (1998) & 0.187 & 0.056 & 0.032 \\
\hline
\end{tabular}

As mentioned above, the procedure was repeated twice, both using the Johnson $B-V$ and the Strömgren $b-y$ colours. Corresponding reddening estimates are provided in Table 3 in units of $E(B-V)$. Values derived from Strömgren $b-y$ colours were converted to $E(B-V)$ using the transformation $E(B-V)=E(b-y) / 0.72$ (Crawford \& Mandwewala 1976). The final reddening adopted for each cluster is the weighted average of the values obtained from the two colours, where errors in the values for each colour are obtained from the dispersion of individual data. These reddenings are $E(B-V)=$ $0.183 \pm 0.005$ for NGC 6397, $E(B-V)=0.040 \pm 0.005$ for NGC 6752, and $E(B-V)=0.024 \pm 0.004$ for 47 Tuc. Systematic uncertainties due to possible offsets between the temperature scales for field and cluster stars caused by errors in individual temperature determinations were evaluated as follows. In total, 40 globular cluster and 35 field stars were used (these include field stars brighter and more evolved than those listed in Table 2). Adopting a common error of $\pm 150 \mathrm{~K}$ for individual temperatures from $\mathrm{H} \alpha$, the possible offset between the two sets is of $\pm 36 \mathrm{~K}$ from simple statistics. This yields zero point errors of $\pm 0.011 \mathrm{mag}$ in the reddening (producing an error of $\pm 0.055 \mathrm{mag}$ in the distance) and of $\pm 0.025 \mathrm{dex}$ in the metal abundance (that in turn transforms into a typical error of $\pm 0.020 \mathrm{mag}$ in distance). However, it should be noted that these two changes in distance are of opposite sign, so that the systematic uncertainty in distance moduli becomes $\pm 0.035 \mathrm{mag}$.

The last two rows of Table 3 list literature reddening determinations taken from the compilation by Harris (1996) and from the maps of interstellar reddening obtained by Schlegel et al. (1998) from analysis of the COBE-DIRBE data. The agreement between the various determinations is excellent, in particular with the latter ones: our values are on average $0.008 \pm$ 0.012 smaller than those listed by Harris, and $0.009 \pm 0.005 \mathrm{mag}$ smaller than Schlegel et al.'s. We note that our reddening values might be systematically smaller than those derived by other authors because we have assumed that the local subdwarfs are unreddened; however, only three of the 35 field stars considered here were found to be reddened by Schuster \& Nissen (1989) and Carney et al. (1994) (namely, HD 116064, HD 132475, and HD 140283); the average reddening of the whole sample is then $E(B-V)=0.003 \mathrm{mag}$. Once this is taken into account, the small residual differences are well within the uncertainties of the various determinations. We in fact estimate that the error on the zero points of our reddenings is $\pm 0.011 \mathrm{mag}$ from the uncertainty in the zero-point of the relative temperature scales. We neglect here the possible impact of the adopted colour-temperature calibration, that we cannot estimate lacking adequate data for $b-y$ colours. The agreement with these independent estimates supports the small error bars we attach to our reddening estimates.

\subsection{Metallicity}

Metal abundances (both $[\mathrm{Fe} / \mathrm{H}]$ values and average overabundance of the $\alpha$-elements [ $\alpha / \mathrm{Fe}]$ ) were derived both for field and clusters stars using strictly the same procedure. The observed field stars cover the metal abundance range from $[\mathrm{Fe} / \mathrm{H}]=$ -0.6 to -2.0 . Within this scale, the three clusters have metal abundances of $[\mathrm{Fe} / \mathrm{H}]=-2.03 \pm 0.05,[\mathrm{Fe} / \mathrm{H}]=-1.43 \pm$ 0.04 , and $[\mathrm{Fe} / \mathrm{H}]=-0.66 \pm 0.04$ for NGC 6397, NGC 6752 and 47 Tuc, respectively. These cluster abundances are quite similar to those obtained by Carretta \& Gratton (1997). It must be noticed that the metal abundances derived for the field stars in the present analysis are lower than those used by Carretta et al. (2000). Discarding HD 145417, the average difference is $0.13 \pm 0.04 \mathrm{dex}$. This difference is due to the lower temperatures adopted in the present analysis. This is the first time that metal abundances consistently derived for field and cluster stars in similar evolutionary phases are used when deriving distances with the Main Sequence Fitting Method.

Figure 1 displays the run of the $B-V$ and $b-y$ colours with metallicity for the field stars in Table 2 at an absolute magnitude $M_{V}=6$. In this figure we have used the overall metallicity $[\mathrm{M} / \mathrm{H}]$. This is connected to $[\mathrm{Fe} / \mathrm{H}]$ by the relation $[\mathrm{M} / \mathrm{H}]=[\mathrm{Fe} / \mathrm{H}]+\log (0.638 f+0.362)$, where $f$ is the average overabundance of the $\alpha$-elements $(\mathrm{Mg}, \mathrm{Si}, \mathrm{Ca}$, and Ti) by number (not in the logarithm; see Straniero et al. 1997). Note that $\log f=0.34,0.29$, and 0.30 for NGC 6397 , NGC 6752, and 47 Tuc respectively (Gratton et al. 2001; Carretta et al. 2003). Appropriate values for the field stars are listed in Table 2. Following Gratton et al. (1997), this comparison was made by correcting the observed colours for the difference due to the absolute magnitude of the stars, by moving the stars parallel to the main sequence. The adopted corrections are $\Delta(B-V)=-\left(M_{V}-6\right)\left[0.192+0.028\left(M_{V}-6\right)\right]$, 


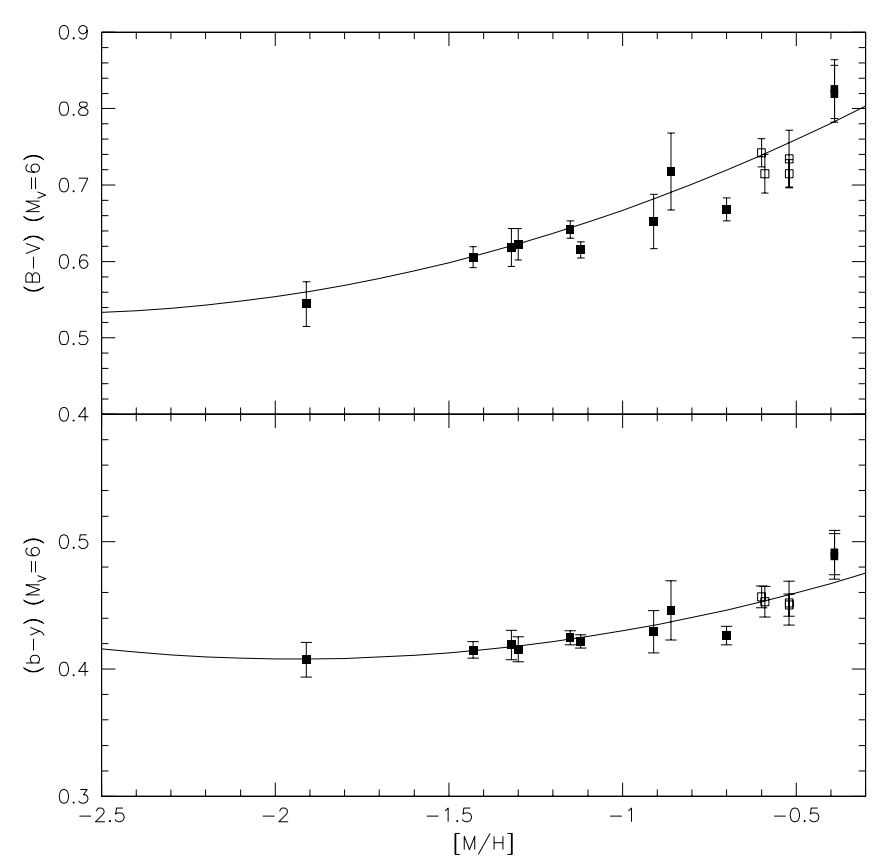

Fig. 1. Colour of the main sequence at an absolute magnitude $M_{V}=6$. The upper panel is for the Johnson $B V$ photometry, the lower panel is for the Strömgren by photometry. Symbols represent individual unevolved subdwarfs (that is, stars with $M_{V}>5.5$ ) with parallax error $\Delta \pi / \pi<0.12$. The lines are the model predictions by Straniero et al. (1997). Filled symbols are the field stars considered in this paper; open squares are additional unevolved subdwarfs, used as a sanity check (see text).

and $\Delta(b-y)=-0.097\left(M_{V}-6\right)$. The lines overimposed to the data in Fig. 1 are not best fit lines, but simply the predictions of the theoretical isochrones by Straniero et al. (1997; as discussed in Gratton et al. 1997 and Carretta et al. 2000 , other isochrone-sets give similar predictions once the same transformations from the theoretical to observational planes are considered). They are represented by the following equations:

$(B-V)_{\left(M_{V}=6\right)}=0.876+0.257[\mathrm{M} / \mathrm{H}]+0.048[\mathrm{M} / \mathrm{H}]^{2}$, and:

$(b-y)_{\left(M_{V}=6\right)}=0.502+0.097[\mathrm{M} / \mathrm{H}]+0.025[\mathrm{M} / \mathrm{H}]^{2}$.

The agreement between theoretical predictions and observations is excellent, although a small offset is present in the Johnson $B-V$ colour. On average, stars are bluer than predicted by models, by $0.014 \pm 0.006 \mathrm{mag}$ in $B-V$ and $0.002 \pm 0.003 \mathrm{mag}$ in $b-y$. These small differences may be ascribed to small uncertainties in the zero point of the photometric pass bands, as well as to an error in the temperature scale of about $30 \mathrm{~K}$. However they have no impact on our distance and age derivation, which only use these equations in a differential way. Once these small offsets are taken into account, the residuals from the theoretical curves agree quite well with observational errors in the parallaxes (the dominant contributor), in the colours, and in the metal abundances: in fact, the reduced $\chi^{2}$ values are 1.53 for $B-V$ and 0.72 for $b-y$. The only star showing a slightly discrepant value is star HD 120559 $([\mathrm{M} / \mathrm{H}]=-0.70)$, that is slightly bluer than expected for its metallicity.
The scatter around the mean relations is rather large for the metal-rich stars. Also our subdwarf sample contains only very few metal-rich stars, thus raising doubts on whether the theoretical relations well predict colours for $[\mathrm{Fe} / \mathrm{H}]>-0.6$. As a sanity check of the colour-metallicity relations at high metal abundances we have considered additional unevolved subdwarfs (i.e. stars with $M_{V}>5.5$ ) taken from the papers of Grundahl et al. (2002) and Percival et al. (2002). There are four stars satisfying our selection criteria (unreddened, bona fide single stars, with complete photometric data and accurate parallaxes). These stars are listed in the lower part of Table 2. Since we do not have spectra for these stars, their metal abundances were obtained by correcting the values listed by Grundahl et al. (2002) for the average offset between the metallicities listed in that paper and the $[\mathrm{Fe} / \mathrm{H}]$ values obtained in our analysis (Gratton et al. 2003) for stars in common between the two samples: this is $0.06 \pm 0.02 \mathrm{dex}$, with our $[\mathrm{Fe} / \mathrm{H}]$ values being smaller. Furthermore, we adopted for the $[\alpha / \mathrm{Fe}]$ values the averages obtained for stars of the appropriate metallicity by Gratton et al. (2003). In this way, colours and metallicities for these stars should be perfectly consistent with those of our program field stars. Data for the additional stars were plotted as open squares in our figures. They agree perfectly with the average relation found for our sample: on average they are bluer than the theoretical predictions by $0.025 \pm 0.008$ mag in $B-V$, and by $0.004 \pm 0.003 \mathrm{mag}$ in $b-y$. Within the errors, these are the same offsets found for the program stars.

The agreement between theoretical predictions and observations is a strong support to the use of colour corrections based on theoretical models when comparing stars and clusters with different metallicities.

Finally we note that the Strömgren $b-y$ colour has a much weaker dependence on metallicity than the Johnson $B-V$ colour, and appears then definitely superior in the analysis of the most metal-poor clusters, for which some extrapolation off the sequence of local subdwarfs is needed.

\subsection{Distances}

Distances to each cluster were obtained by fitting the observed globular cluster mean loci to the location of the local subdwarfs in the colour magnitude diagram. Only the bona fide single subdwarfs with absolute magnitude $M_{V}>5.5$ listed in the upper portion of Table 2 were used to fit each cluster locus, to avoid objects possibly evolved off the main sequence. Colours for each field star were corrected for the difference in metallicity between the star and the clusters, using the above theoretical relations (1) and (2), from Straniero et al. (1997) isochrones. Distances obtained from each individual subdwarf were averaged weighting them according to their errors. These were the sum in quadrature of the uncertainties in the absolute magnitude due to errors in the parallaxes, and of the uncertainties due to errors in colours (assumed to be $0.01 \mathrm{mag}$ in $B-V$, $0.007 \mathrm{mag}$ in $b-y$ ) and metallicity (assumed to be $\pm 0.04 \mathrm{dex}$ ).

Derived distance moduli were then shortened by $0.02 \mathrm{mag}$ to account for the possible presence of undetected binaries; this 
Table 4. Distances and ages for globular clusters.

\begin{tabular}{lccc}
\hline \hline Parameter & NGC 6397 & NGC 6752 & \multicolumn{1}{c}{47 Tuc } \\
\hline$[\mathrm{Fe} / \mathrm{H}]$ & $-2.03 \pm 0.05$ & $-1.43 \pm 0.04$ & $-0.66 \pm 0.04$ \\
{$[\alpha / \mathrm{Fe}]$} & $0.34 \pm 0.02$ & $0.29 \pm 0.02$ & $0.30 \pm 0.02$ \\
{$[\mathrm{M} / \mathrm{H}]$} & $-1.79 \pm 0.04$ & $-1.22 \pm 0.04$ & $-0.45 \pm 0.04$ \\
& & & \\
$(m-M)_{V(B-V)}$ & $12.57 \pm 0.03$ & $13.38 \pm 0.03$ & $13.47 \pm 0.03$ \\
$(m-M)_{V(b-y)}$ & $12.62 \pm 0.03$ & $13.14 \pm 0.03$ & $13.57 \pm 0.03$ \\
$<(m-M)_{V}>$ & $12.60 \pm 0.08$ & $13.26 \pm 0.08$ & $13.52 \pm 0.08$ \\
bin. corrected & $12.58 \pm 0.08$ & $13.24 \pm 0.08$ & $13.50 \pm 0.08$ \\
& & & \\
$V(\mathrm{TO})$ & $16.56 \pm 0.02$ & $17.39 \pm 0.03$ & $17.68 \pm 0.05$ \\
$V(\mathrm{HB})$ & $13.11 \pm 0.10$ & $13.84 \pm 0.10$ & $14.13 \pm 0.10$ \\
$M_{V}(\mathrm{TO})$ & $3.98 \pm 0.08$ & $4.15 \pm 0.08$ & $4.18 \pm 0.08$ \\
$M_{V}(\mathrm{HB})$ & $0.53 \pm 0.13$ & $0.60 \pm 0.13$ & $0.63 \pm 0.13$ \\
& & & \\
Age (no diff.) $(\mathrm{Gyr})$ & $13.9 \pm 1.1$ & $13.8 \pm 1.1$ & $11.2 \pm 1.1$ \\
Age (diff.) $(\mathrm{Gyr})$ & $13.5 \pm 1.1$ & $13.4 \pm 1.1$ & $10.8 \pm 1.1$ \\
\hline
\end{tabular}

small correction is the same adopted by Carretta et al. (2000). Relevant data and results are given in Table 4.

Two different estimates of the distance to each cluster were obtained in this way, corresponding to fitting the $V-(B-V)$ diagram in the Johnson system, and the $V-(b-y)$ diagram in the Strömgren system, respectively. The attached error is the fitting error produced by the individual star errors. Figure 2 displays the fits we obtained for the three clusters in each colour. Adopted distances are the simple averages of the values obtained with each colour. There is no significant systematic difference between distances obtained from Johnson and Strömgren photometry, the average difference being $0.03 \pm 0.12 \mathrm{mag}$. The errors expected from uncertainties in data for individual stars are actually much smaller (about $0.025 \mathrm{mag}$ ). The worst case is that of NGC 6752: in this case the distance moduli given by $B-V$ is 0.24 mag larger than that given by $b-y$, a difference much larger than the internal errors of the fits. Adopted metallicities and reddenings are unlikely to be the cause for this difference - e.g. a metallicity as low as $[\mathrm{M} / \mathrm{H}]=-1.8$ (corresponding to $[\mathrm{Fe} / \mathrm{H} \sim-2$ ) is required to cancel it out; an error of 0.1 dex (yielding a metal abundance coincident with that estimated by Kraft \& Ivans 2003, for this cluster) may only justify a difference of about $0.04 \mathrm{mag}$. On the other side, a part of the difference is due to the use of a unique reddening value in both determinations: if we had rather used the reddening estimates obtained separately for each colour (thus taking into account possible zero-point offsets in the colour calibrations), the difference would have been reduced to $0.18 \mathrm{mag}$. We think that the residual difference is mainly due to inaccuracies in the colour term of the photometric transformations from one system to the other. This is also supported by some small mismatches between the globular cluster mean loci and the location of the local subdwarfs, visible in various panels of Fig. 2. These have impact on the distance derivations because the stars used to derive distances are redder than those used to derive reddening and metal abundances. However, we cannot judge from our photometry alone which of the two results - from $B-V$ or from $b-y$, is correct. Luckily, differences are much smaller for the two other clusters. Averaging data for the three clusters, we then estimated that the internal errors in the distance moduli are \pm 0.074 mag.

We note that distances do not change if the four additional stars used as sanity check (see Sect. 4.2) are used in the fittings.

Systematic errors in these distance estimates are mainly due to the zero point of our effective temperatures; as discussed in Sect. 4.1 they are of about \pm 0.04 mag. The final error bars of our distance moduli were obtained by summing quadratically these two contributions.

A small systematic error in our distances is actually related to uncertainties in the metallicity scale, due to the quadratic form of the metallicity-colour relations. Our metallicities for these three clusters are very similar to those obtained very recently by Kraft \& Ivans (2003), using analysis of giant stars with different model atmospheres: our $[\mathrm{Fe} / \mathrm{H}]$ values are $0.05 \pm 0.06$ dex higher than those obtained by them using the MARCS model atmospheres, and $0.01 \pm 0.04$ dex lower than those obtained with the Kurucz model atmospheres (using Fe II lines, their preferred abundance estimators). However, Kraft \& Ivans conclude that various sources of possible errors make this scale uncertain by at least $0.03-0.05 \mathrm{dex}$. A similar result is obtained by comparing our abundances for the field stars with other recent studies (see Table 10 of Gratton et al. 2003). To estimate the impact of the uncertainty in the metallicity scale on our distance derivations, we repeated them by arbitrarily increasing the metallicities for both field and cluster stars by 0.1 dex (likely, an estimate of the maximum zero point errors in our metallicities). We found that the distance modulus to NGC 6397 would be reduced by $0.04 \mathrm{mag}$, that to NGC 6752 


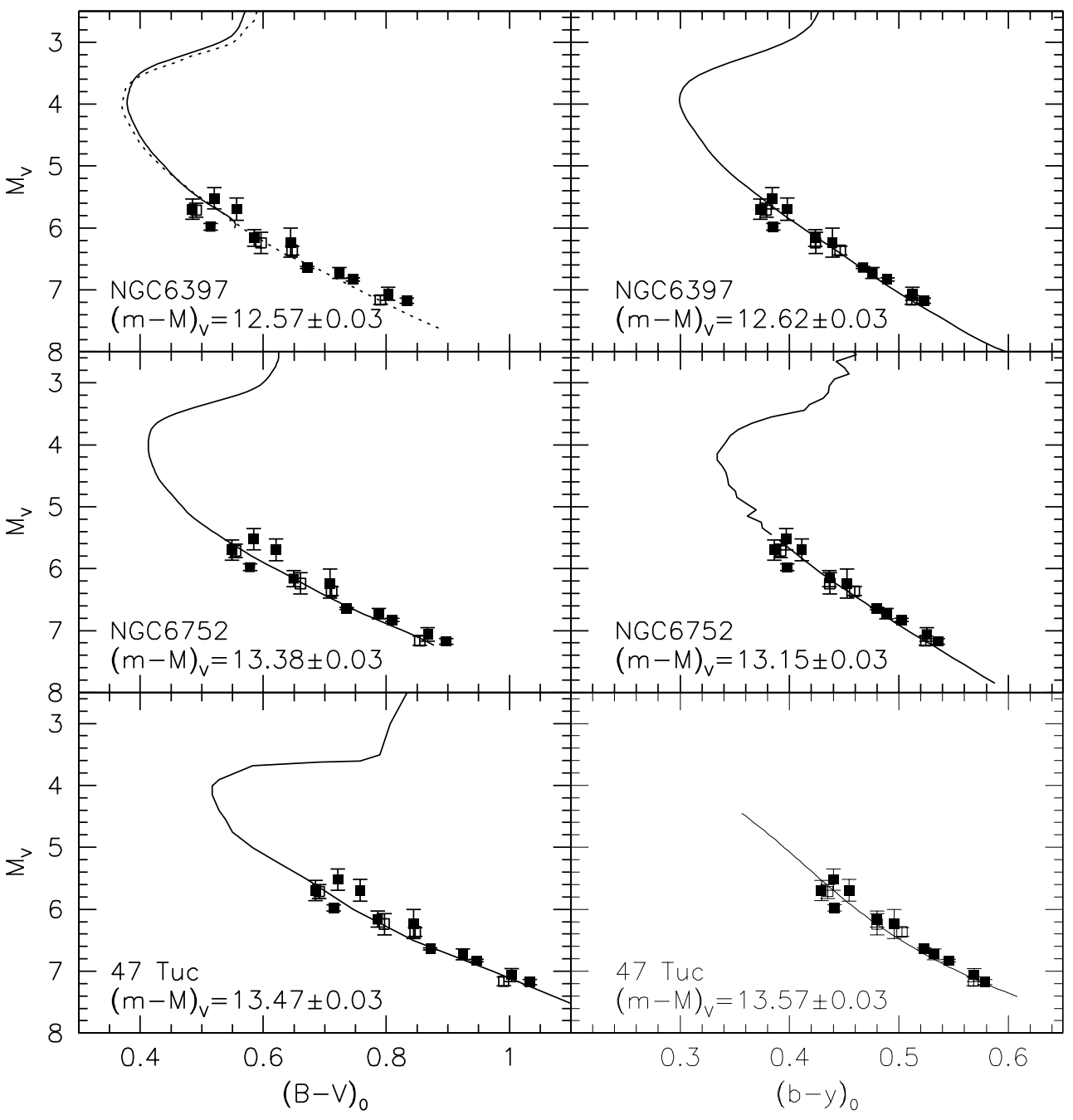

Fig. 2. Main Sequence Fitting distances to the program clusters (NGC 6397: upper panels; NGC 6752: middle panels; 47 Tuc: lower panels). For each cluster fits obtained both from Johnson $B-V$ (left) and Strömgren $b-y$ (right) colours are shown. The parameters adopted in these fits are listed in Tables 2 and 3. Data for individual bona fide single subdwarfs are shown with their error bars. Solid and dashed lines in the upper left panel are the cluster mean loci from Kaluzny (1997) and Alcaino et al. (1997), respectively. Filled symbols are the subdwarfs used to estimate the distance moduli (upper portion of Table 2); open squares are additional unevolved subdwarfs, used as a sanity check (see text).

by $0.01 \mathrm{mag}$, and finally that to 47 Tucanae would be increased by $0.03 \mathrm{mag}$. While not negligible, this source of errors appears to be much smaller than that due to the reddening and photometric errors.

\subsection{Comparison with previous determinations}

The literature on distance determinations to the program clusters is very extensive and here we only consider a few results. Table 5 compares the present distances with previous estimates obtained using the MSF technique. On average, our new distances are shorter: differences are $0.16 \pm 0.06 \mathrm{mag}$ and $0.08 \pm 0.01$ with Reid (1998) and Carretta et al. (2000), respectively ${ }^{2}$. While the source of parallaxes for the local subdwarfs in all these various analyses is always the Hipparcos catalogue,

2 A downward correction to MSF distances by about 0.1 mag was already suggested by Carretta et al. (2000) based on a comparison of distances derived by this technique with other values. This difference was there attributed to small inconsistencies in the reddening and a difference of our study is that we used both the Johnson and Strömgren photometry, and we also made different assumptions about metallicities and reddenings for both cluster and field stars (values adopted for the clusters in each analysis are listed in the second and third columns of Table 5).

Differences between the various distance estimates are large for 47 Tuc, with values spanning a range of $0.35 \mathrm{mag}$ (our new determination is in the middle of the other values). To understand these differences, first we notice that Reid (1998) and Carretta et al. (2000) used the Hesser et al. (1987) BV photometry without any correction, while we have corrected the 47 Tuc photometry according to Percival et al. (2002). All other terms held constant, this causes the distance modulus of this cluster to be 0.19 mag smaller. Furthermore, while the

metallicity scales for globular cluster and local subdwarfs. This correction is confirmed both in sign and size by the present analysis that uses a new homogeneous evaluation of reddenings and metallicities for globular clusters. Indeed, our distance scale is very similar to that final one adopted by Carretta et al. (2000). 
Table 5. Comparison with previous distance determinations using the Main Sequence Fitting method based on Hipparcos parallaxes for the local subdwarfs. Errors quoted are those given in the original papers; they have different meanings from the present error bar.

\begin{tabular}{lccc}
\hline \hline Author & {$[\mathrm{Fe} / \mathrm{H}]$} & $E(B-V)$ & $(m-M)_{V}$ \\
\hline \multicolumn{4}{c}{ NGC 6397 } \\
Reid (1998) & -1.82 & 0.19 & $12.83 \pm 0.15$ \\
This paper & -2.03 & 0.183 & $12.58 \pm 0.08$ \\
& NGC 6752 & & \\
Reid (1998) & -1.42 & 0.04 & $13.28 \pm 0.15$ \\
Carretta et al. (2000) & -1.43 & 0.035 & $13.32 \pm 0.04$ \\
This paper & -1.43 & 0.040 & $13.24 \pm 0.08$ \\
& 47 Tuc & & \\
Reid (1998) & -0.70 & 0.04 & $13.68 \pm 0.15$ \\
Carretta et al. (2000) & -0.67 & 0.055 & $13.57 \pm 0.09$ \\
Percival et al. (2002) & -0.7 & 0.04 & $13.37 \pm 0.11$ \\
Grundahl et al. (2002) & -0.67 & 0.04 & $13.33 \pm 0.10$ \\
This paper & -0.66 & 0.024 & $13.50 \pm 0.08$ \\
\hline
\end{tabular}

cluster Fe abundance adopted in the various analyses appears to be quite similar, there are significant differences in the abundances adopted for the comparison field stars. Reid (1998), Carretta et al. (2000), and Percival et al. (2002) all adopt the same metal abundances, essentially consistent with the scale defined by Carretta et al. (2000). However, as we already mentioned, this is 0.13 dex higher than that adopted here, that is obtained from an analysis which is fully consistent for field and cluster stars. At the metallicity of 47 Tuc, this corresponds to a difference of $0.25 \mathrm{mag}$ in the distance modulus, with the present one being longer. Finally, the reddening adopted here (for the first time, consistently derived for field and cluster stars) is 0.031 mag smaller than that adopted by Carretta et al. (2000) and Percival et al. (2002), and 0.016 mag smaller than that considered by Reid (1998). This causes our distance modulus to be 0.15 mag shorter than that in Carretta et al. (2000) and Percival et al. (2002), and 0.08 mag shorter than that in Reid (1998). When comparing our results with those of Grundahl et al. (2002), it should be first noticed that different colours are used (we use the $b-y$ colour, while Grundahl et al. use the $v-y$ colour). Furthermore, there is a difference in the adopted reddening, and again in the metal abundance of the field stars (their finally adopted values being larger than ours by $0.16 \mathrm{dex}$, because they corrected upward by 0.1 dex the abundances originally obtained for the field stars in order to reach a claimed consistency with the value obtained for the cluster).

These different assumptions about photometry, metal abundances and reddenings fully explain the differences between our new determinations and the above literature values: they also emphasize the importance of consistent derivations of metal abundances and reddenings for field and cluster stars.

Differences are smaller for NGC 6752; they again may be explained by the different assumptions about metal abundances and reddenings. In the case of NGC 6397 (not included in the
Table 6. Comparison between our distance determinations and those from the White Dwarf cooling sequence.

\begin{tabular}{lcc}
\hline \hline Author & $E(B-V)$ & $(m-M)_{V}$ \\
\hline \multicolumn{3}{c}{ NGC 6752} \\
Renzini et al. (1996) & 0.04 & $13.17 \pm 0.13$ \\
This paper & 0.040 & $13.24 \pm 0.08$ \\
& Tuc & \\
Zoccali et al. (2001) & 0.055 & $13.27 \pm 0.13$ \\
This paper & 0.024 & $13.50 \pm 0.08$ \\
\hline
\end{tabular}

sample considered by Carretta et al. 2000), we have used a metallicity $\sim 0.2$ dex lower (this causes a 0.12 mag shortening of the distance modulus), and a reddening about 0.007 mag smaller (that translates into a distance modulus 0.04 mag shorter) than those adopted by Reid (1998), and this explains about two thirds of the 0.25 mag difference found in the distance moduli for this cluster.

Among the various other distance determinations, we considered only those obtained from fitting the white dwarf cooling sequence observed in globular clusters with a sequence of local white dwarfs with accurate parallaxes. This comparison is shown in Table 6. Distances from the white dwarf cooling sequence are independent of metallicity (at least as long as the assumption that the white dwarf masses do not depend on metallicity is correct); however they have a dependence on interstellar reddening quite similar to that obtained for the MSF method, because the two sequences are roughly parallel in the colour-magnitude diagram. We found good agreement with the results of Renzini et al. (1996) for NGC 6752, while the agreement is far from being satisfactory for 47 Tuc, whose distance modulus from the white dwarf cooling sequence (Zoccali et al. 2001) is 0.23 mag shorter than our MSF value (see last column of Table 6). Indeed the difference is even larger: in fact, if the same reddening values were adopted, the offset would be as large as $0.38 \mathrm{mag}$. The reason for such a large discrepancy between these two distance determinations for this cluster remains unexplained.

\section{Luminosity of the horizontal branch}

\subsection{Derivation of the absolute luminosity of the $H B$}

Once the distance of a cluster is known, derivation of the absolute luminosity of the $\mathrm{HB}, M_{V}(\mathrm{HB})$, from the observed HB apparent luminosity, $V(\mathrm{HB})$, seems quite straightforward. $M_{V}(\mathrm{HB})$ is usually defined as the $V$ absolute magnitude of an HB star at $\log T_{\text {eff }}=3.85$, that is taken to represent the equilibrium temperature of an RR Lyrae star near the middle of the instability strip. However, since the HB is not exactly horizontal, not even in the $V$ band, and since the three clusters considered in the present paper lack a significant population of RR Lyrae stars, the estimate of their HBs average luminosities is not so easy. Furthermore, care should be devoted to ensure that photometry of both $\mathrm{HB}$ and main sequence stars in the cluster are on the same scales. To ensure 
proper handling of these issues, we adopted simply the magnitude difference between TO and HB measured for the three clusters by Rosenberg et al. (1999), who carefully considered these points. By combining Rosenberg et al.'s values with our TO absolute magnitudes (see Sect. 6.1), we obtained HB absolute magnitudes of $0.53 \pm 0.13,0.60 \pm 0.13$, and $0.63 \pm 0.13$ for NGC 6397, NGC 6752, and 47 Tuc, respectively (see Table 4). The error bars attached to these $M_{V}(\mathrm{HB})$ 's were obtained by combining those of Rosenberg et al. (1999), with the errors in our estimates of the absolute magnitude of the TO.

The three values may be averaged together by adopting a relation between the absolute magnitude of the HB and metallicity. This is generally described in terms of a linear relation. However, claims have been made that the relation is not strictly linear (Caputo et al. 2000; Rey et al. 2000, and references therein) and doubts have been cast on whether a universal luminosity-metallicity relations holds for HB stars in different environments, e.g. the MW field versus cluster stars, and the MW versus other Local Group galaxies. Indeed, even at fixed metallicity there is an intrinsic spread in the HB luminosity due to evolutionary effects whose extent varies as a function of metal abundance (Sandage 1990), and it has been shown that the luminosity-metallicity relation may not be strictly linear because it depends also on the HB morphology and stellar population (Caputo et al. 2000; Demarque et al. 2000). In principle, since our three clusters span a range in metallicity of about $1.4 \mathrm{dex}$, their $M_{V}(\mathrm{HB})$ values could be used to derive the slope of the luminosity-metallicity relation for Galactic globular clusters. However, because of the very small number of clusters and the still rather large error bars of the $M_{V}(\mathrm{HB})$ values, the result is very uncertain: $\Delta M_{V}(\mathrm{HB}) / \Delta[\mathrm{Fe} / \mathrm{H}]=0.07 \pm$ $0.13 \mathrm{mag} \mathrm{dex}^{-1}$. The definition of the slope of the luminositymetallicity relation for $\mathrm{HB}$ stars is beyond the purposes of the present paper; to correct the $M_{V}(\mathrm{HB})$ values of our three clusters to a common metallicity we use literature results. A quite accurate and robust estimate for the slope has been recently obtained by Clementini et al. (2003), based on observations of a hundred RR Lyrae stars in the LMC. The slope obtained in that paper $\left(0.21 \pm 0.05 \mathrm{mag} \mathrm{dex}^{-1}\right)$ is in very good agreement with that been determined by Rich et al. (2002; $0.22 \mathrm{mag} \mathrm{dex}^{-1}$ ) from the mean HB magnitudes at the middle of the instability strip of 19 globular clusters in M31; it also agrees with the slope found by the Baade-Wesselink analysis of the MW field RR Lyrae stars $\left(0.20 \pm 0.04\right.$ mag dex $^{-1}$ : Fernley et al. 1998). Theoretical models of helium-burning stars also predict the slope of the luminosity metallicity relation for HB stars ${ }^{3}$. Several uncertainties affect the theoretical models, that explain the scatter observed among different families of HB models. However, all the most recent

\footnotetext{
3 Actually, theoretical models predict the luminosity of the Zero Age Horizontal Branch (ZAHB) and its dependence on metallicity. The correction of the theoretical $M_{V}(\mathrm{ZAHB})-[\mathrm{Fe} / \mathrm{H}]$ relation to the observed $M_{V}(\mathrm{HB})-[\mathrm{Fe} / \mathrm{H}]$ relation is made either applying a fixed offset that takes into account evolution (generally of the order of 0.08-0.10 mag) or using an empirical correction as the one derived by Sandage $(1993) \Delta V(\mathrm{ZAHB}-\mathrm{HB})=0.05[\mathrm{Fe} / \mathrm{H}]+0.16$. This relation corresponds to an evolutionary correction of about $0.085 \mathrm{mag}$ at $[\mathrm{Fe} / \mathrm{H}]=-1.5$.
}

Table 7. Comparison between various determinations of the luminosity of the horizontal branch (from Cacciari \& Clementini 2003).

\begin{tabular}{lc}
\hline \hline Method & $\begin{array}{c}M_{V}(\mathrm{RR}) \\
\text { at }[\mathrm{Fe} / \mathrm{H}]=-1.5\end{array}$ \\
\hline Statistical parallaxes & $0.78 \pm 0.12$ \\
Trigonometric parallaxes (RR Lyr) & $0.58 \pm 0.13$ \\
Trigonometric parallaxes (HB stars) & $0.62 \pm 0.11$ \\
Baade-Wesselink (RR Cet) & $0.55 \pm 0.12$ \\
HB stars: Evolutionary models - bright & $0.43 \pm 0.12$ \\
HB stars: Evolutionary models - faint & $0.56 \pm 0.12$ \\
Pulsation models (visual) & $0.58 \pm 0.12$ \\
Pulsation models ( $\left.P L_{\mathrm{K}} Z\right)$ & $0.59 \pm 0.10$ \\
Pulsation models (double-modes) & $0.57 \pm 0.06$ \\
Fourier parameters & $0.61 \pm 0.05$ \\
\hline Main Sequence Fitting & $0.56 \pm 0.07$ \\
\hline
\end{tabular}

models (Caloi et al. 1997; Cassisi et al. 1999; Ferraro et al. 1999; Demarque et al. 2000; VandenBerg et al. 2000) agree that although the relation is not universal and not strictly linear, it can be roughly described by a linear relation with average slope $\sim 0.23$, as a first approximation. We adopted the value $\Delta M_{V}(\mathrm{HB}) / \Delta[\mathrm{Fe} / \mathrm{H}]=0.22 \mathrm{mag} \mathrm{dex}^{-1}$ as the average of the above independent estimates and attached to this slope a conservative error of $\pm 0.05 \mathrm{mag} \mathrm{dex}^{-1}$ (Clementini et al. 2003). We derived $M_{V}(\mathrm{HB})=0.65 \pm 0.13,0.59 \pm 0.13$, and $0.45 \pm 0.13$ for NGC 6397, NGC 6752, and 47 Tuc respectively, giving a zero point of $0.56 \pm 0.07$ at $[\mathrm{Fe} / \mathrm{H}]=-1.5$. The error bar is obtained combining the internal errors of the values obtained for each cluster. The relation between absolute magnitude of the $\mathrm{HB}$ and metallicity adopted from our analysis of the three clusters is then:

$M_{V}(\mathrm{HB})=(0.22 \pm 0.05)([\mathrm{Fe} / \mathrm{H}]+1.5)+(0.56 \pm 0.07)$

\subsection{Comparison with other determinations and implications: The distance to the LMC}

The zero point of the absolute magnitude-metallicity relation has been determined by a large variety of methods and approaches; a recent, comprehensive review is provided by Cacciari \& Clementini (2003). In their Table 2, that we reproduce in our Table 7 for ease of comparison, these authors provide a summary of determinations of $M_{V}(\mathrm{RR})$ at $[\mathrm{Fe} / \mathrm{H}]=$ -1.5 obtained by several independent methods. Our zero point in Eq. (3) (see last row of Table 7) agrees with the results from the trigonometric parallax of RR Lyrae itself $M_{V}(\mathrm{RR})=0.61 \pm$ 0.11 (Benedict et al. 2002), at $[\mathrm{Fe} / \mathrm{H}]=-1.4$ (Clementini et al. 1995), and with Koen \& Laney (1998) re-evaluation of Gratton (1998) trigonometric parallaxes for several HB stars: $M_{V}(\mathrm{RR})=0.62 \pm 0.11$ at $[\mathrm{Fe} / \mathrm{H}]=-1.5$. Very good agreement also exists with Cacciari et al. (2000) revision of the BaadeWesselink absolute magnitude of the RR Lyrae star RR Ceti: $M_{V}(\mathrm{RR})=0.55 \pm 0.12$ at $[\mathrm{Fe} / \mathrm{H}]=-1.5$, and with the 
prediction both of the pulsation models and of the Fourier parameters (see Table 7 and discussion in Cacciari \& Clementini 2003). Concerning the theoretical models, our $M_{V}(\mathrm{HB})$ is in excellent agreement with the family of models that predict a faint value of $M_{V}(\mathrm{RR})=0.56 \pm 0.12$, namely Ferraro et al. (1999), Straniero et al. (1997), Demarque et al. (2000), and VandenBerg et al. (2000) models. Agreement is less satisfactory, but still within the error bars, with the models by Caloi et al. (1997) and Cassisi et al. (1999) who predict a brighter zero point of $M_{V}(\mathrm{RR})=0.43 \pm 0.12$. The statistical parallaxes is the only technique that disagrees and gives a value about 0.2 mag fainter than the present $M_{V}(\mathrm{HB})$. Cacciari \& Clementini (2003) discuss in detail the problems connected to this technique and conclude that a more accurate and detailed modeling of the stellar motions in the Galaxy and larger sample of stars are needed to provide reliable and robust results.

Clementini et al. (2003) have recently derived a very accurate estimate of the average luminosity of the RR Lyrae variables in the bar of the LMC: $V_{0}(\mathrm{RR})=19.064 \pm 0.064$ at $[\mathrm{Fe} / \mathrm{H}]=-1.5$. Combined with our new estimate of the absolute luminosity of the HB, this leads to a distance modulus to the LMC of $(m-M)_{0}=18.50 \pm 0.09 \mathrm{mag}$, in perfect agreement with the value obtained by Clementini et al. (2003).

\section{Ages}

\subsection{Derivation of ages}

Once distances and metallicities are known, ages can be derived from the luminosity of the TO. Relevant values are listed in Table 4. We found the literature estimates of the apparent magnitude of the TO point rather unsatisfactory for these three clusters. Therefore, we redetermined them using a polynomial fit of the mean loci in the TO region. Parabolic fits were used for NGC 6397 and NGC 6752, and a cubic fit for 47 Tuc. Again, we repeated the procedure both for the $V,(B-V)$ and the $V,(b-y)$ diagrams, and averaged the results. We attached to these average values errors in the range 0.02-0.05 mag, as estimated from the difference of the two determinations. Absolute magnitudes of the TO of the three clusters were then derived by simply combining our distance estimates with the apparent magnitude of the TO. Finally, ages $t$ were estimated by entering the values of the absolute magnitude of the TO, along with the cluster metallicity corrected for the $\alpha$-element overabundance, in the equation $\log t=0.425 M_{V}-$ $0.132[\mathrm{M} / \mathrm{H}]-0.776$ derived by Straniero et al. (1997). This formula is based on standard isochrones, computed with no consideration of sedimentation due to microscopic diffusion (see below). Note that the Straniero et al. (1997) isochrones were computed assuming an helium content of $Y=0.23$. Recent cosmological data rather suggests a slightly larger value of $Y \sim 0.245$ (see e.g. Spergel et al. 2003), in agreement with determinations for globular clusters based on the so-called $R$-method (Cassisi et al. 2003). Adopting such a higher helium content would result in ages about $0.3 \mathrm{Gyr}$ younger (see e.g. D'Antona et al. 2002). We then corrected our age estimate for this effect, that is about $1 / 5$ of our final error bar on the absolute ages for the globular clusters.
Table 8. Model error budget.

\begin{tabular}{lcc}
\hline \hline Error source & Distribution & $\begin{array}{c}\text { Limits } \\
(\mathrm{Gyr})\end{array}$ \\
\hline Convection & Flat & $-0.4,0.4$ \\
Code & Flat & $-0.4,0.4$ \\
Micr. Diffusion & Gaussian & 0.4 at -0.4 \\
Solar $M_{V}$ & Flat & $-0.3,0.3$ \\
\hline
\end{tabular}

The ages we determined for the three clusters are $13.9 \pm 1.1$, $13.8 \pm 1.1$, and 11.2 \pm 1.1 Gyr for NGC 6397, NGC 6752, and 47 Tuc respectively. The uncertainties were obtained by error propagation from the errors in the distance derivations and in the metallicities, and do not take into account the systematics of residual differences in the adopted temperature scales for field and cluster stars (see Sect. 3.2), and of adopted stellar models. The former contribute about $0.4 \mathrm{Gyr}$ to the uncertainty in ages. To estimate the contribution of the models, we used a MonteCarlo approach similar to that described by Carretta et al. (2000). A more rigorous version of this approach was presented by Chaboyer et al. (1996), who obtained very similar results ${ }^{4}$. We considered several sources of theoretical uncertainties (treatment of convection and microscopic diffusion, code, normalization to the Solar absolute magnitude) and assumed a suitable distribution for the impact on ages of each of these contributors. We then combined the individual errors in random extractions, and estimated the standard deviation of the final distribution. This value was then attached to the observed value as representative of theoretical uncertainties. Shapes and limits of the adopted distribution for each error source are given in Table 8.

We note here that the impact of microscopic diffusion is strongly constrained by our results for NGC 6397 (Gratton et al. 2001): they clearly disagree with results obtained by several authors, assuming complete ionization (see Chaboyer et al. 2002). A careful discussion of this topic is given by Richard et al. (2002), who presented results from more sophisticated models that account for partial ionization and the effect of levitation due to radiation pressure. However, also the predictions from these more advanced models do not agree with the results of Gratton et al. (2001), unless it is arbitrarily assumed that there is some mixing at the base of the outer convective envelope, possibly induced by some turbulence. Once this is considered, the impact of microscopic diffusion is constrained to be at most half of the value predicted by models with complete ionization, that is not larger than about 0.5 Gyr. In our estimates we have assumed that uncertainties in the treatment of microscopic diffusion may be represented by a Gaussian with a standard deviation of $\pm 0.4 \mathrm{Gyr}$, centered at a value of $-0.4 \mathrm{Gyr}$, since some small impact of microscopic diffusion is still expected, even if turbulence is present (see Chaboyer et al. 2002;

\footnotetext{
4 The main difference between the two approaches is that contribution by the various sources of errors are assumed to be uncorrelated in the analysis of Carretta et al., while possible correlations are included in the approach of Chaboyer et al.
} 


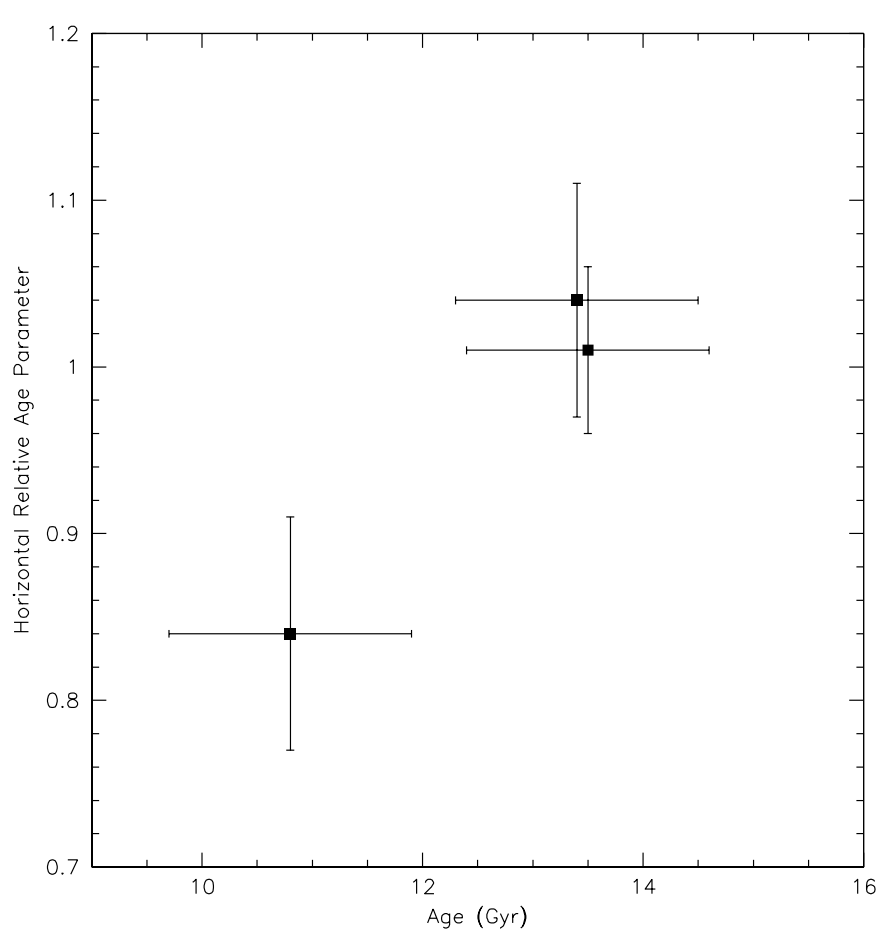

Fig. 3. Comparison between our absolute ages for the program clusters and the relative age determined by Rosenberg et al. (1999) with the horizontal method.

VandenBerg et al. 2002). From this approach we conclude that the theoretical error bar is $0.6 \mathrm{Gyr}$, and that a downward correction of 0.4 Gyr should be applied to the ages derived from isochrones that do not include sedimentation due to microscopic diffusion.

Our data suggests that 47 Tuc may be younger than NGC 6397 and NGC 6752. The age difference (2.6 Gyr) is slightly larger than the sum of the error bars. A similar age difference between these clusters was obtained by Rosenberg et al. (1999) and Salaris \& Weiss (2002) from differential age determinations. Figure 3 compares relative and absolute age estimates for the three clusters. The suggestion that 47 Tuc is indeed younger than the two other clusters is strong, although perhaps not yet conclusive. It seems thus wiser not to include 47 Tuc when determining the age of the oldest clusters in our Galaxy. On the other hand, these same studies also provide strong evidences that both NGC 6397 and NGC 6752 are nearly coeval to the oldest clusters in the Galaxy. The average age for these two clusters (corrected downward by $0.4 \mathrm{Gyr}$ as discussed above) is then $13.4 \pm 0.8 \pm 0.6 \mathrm{Gyr}$; we will assume that this is the age of the oldest Galactic globular clusters. 47 Tuc (with other disk clusters) is likely about $2.6 \mathrm{Gyr}$ younger.

Our age estimate for the oldest globular clusters agrees well with the other evaluations mentioned in the Introduction. It is nearly coincident with the value of $14.0 \pm 2.4$ Gyr determined in a completely independent way using nucleocosmochronology for the extremely metal-poor star CS 31082-001 by Hill et al. (2002). It agrees also fairly well with the age determined for M 4 by Hansen et al. (2002). However, in view of the large uncertainties present in both these determinations, this agreement may be fortuitous. Finally, our age of the oldest

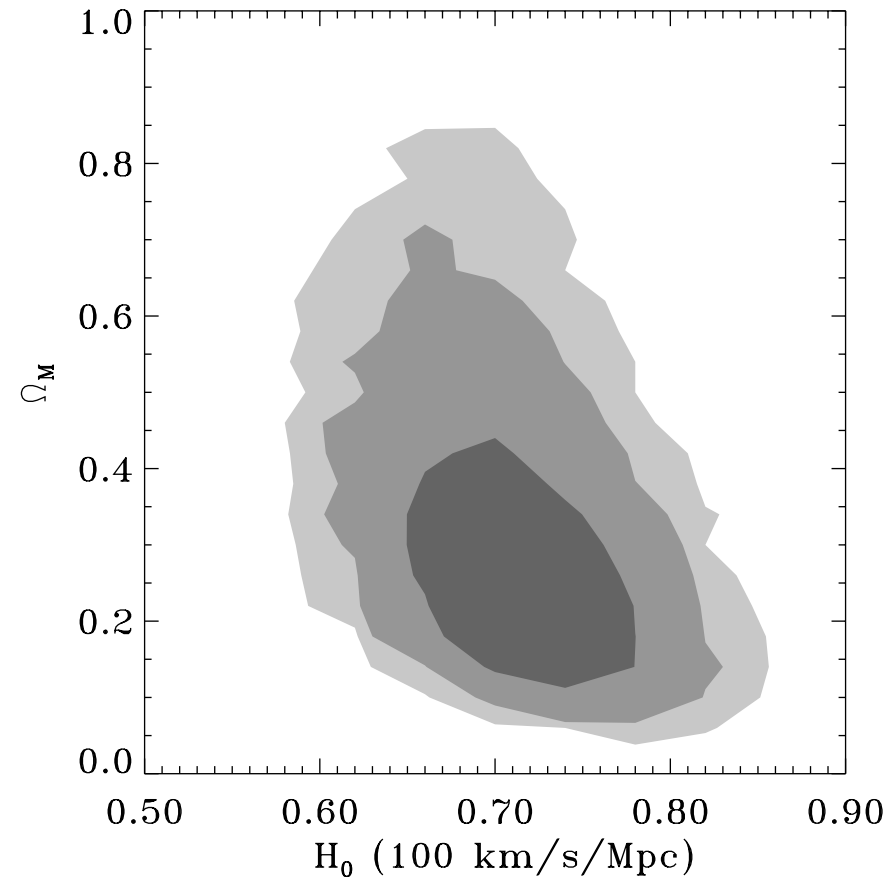

Fig. 4. Values of the matter density parameter $\Omega_{\mathrm{M}}$ as a function of the Hubble constant $H_{0}$ in a flat Universe, obtained from a 10000 random extraction of the values of the age of the Universe, assumed to be $0.3 \mathrm{Gyr}$ older than the age of the oldest globular clusters (13.4 $\pm 1.4 \mathrm{Gyr}$ ), and of $H_{0}$ determined by Spergel et al. (2003) from WMAP experiment and by the HST Key-Project (Freedman et al. 2001): $71 \pm 4 \mathrm{~km} \mathrm{~s}^{-1} \mathrm{Mpc}^{-1}$. Different gray areas correspond to 67 , 95 and $99 \%$ levels of confidence.

globular clusters coincides with the determination of the age of the Universe by the WMAP group (Spergel et al. 2003).

\subsection{The age of the Galaxy in a cosmological framework}

Our estimate of $13.4 \pm 0.8 \pm 0.6$ Gyr for the age of the oldest globular clusters provides useful constraints for cosmology. Since the Universe must be older than the globular clusters, its age is constrained to be larger than $12.0 \mathrm{Gyr}$ at $1-\sigma$ level, and larger than $10.6 \mathrm{Gyr}$ at a $2-\sigma$ level. On the other hand, the WMAP data (Spergel et al. 2003) and the HST key-project results (Freedman et al. 2001) constrain the value of $H_{0}$ at $71 \pm 4 \mathrm{~km} \mathrm{~s}^{-1} \mathrm{Mpc}^{-1}$ (Spergel et al. 2003). Assuming that the Universe is 0.3 Gyr older than the oldest globular clusters, by performing integration of the appropriate equation using 10000 values of the age of the Universe and of $H_{0}$ randomly extracted around these values (see Fig. 4), we found that the value of $\Omega_{\mathrm{M}}$ in a flat Universe (as indicated by the microwave background data: see Spergel et al. 2003) is constrained to be $\Omega_{\mathrm{M}}<0.57$ and $\Omega_{\mathrm{M}}<0.75$ at $95 \%$ and $99 \%$ level of confidence, respectively. This result indicates the strong need for a dark energy even without taking into account the results obtained from the high redshift type Ia SNe (Perlmutter et al. 1999), and those given by galaxy clusters (see e.g. the review by Rosati et al. 2002). 


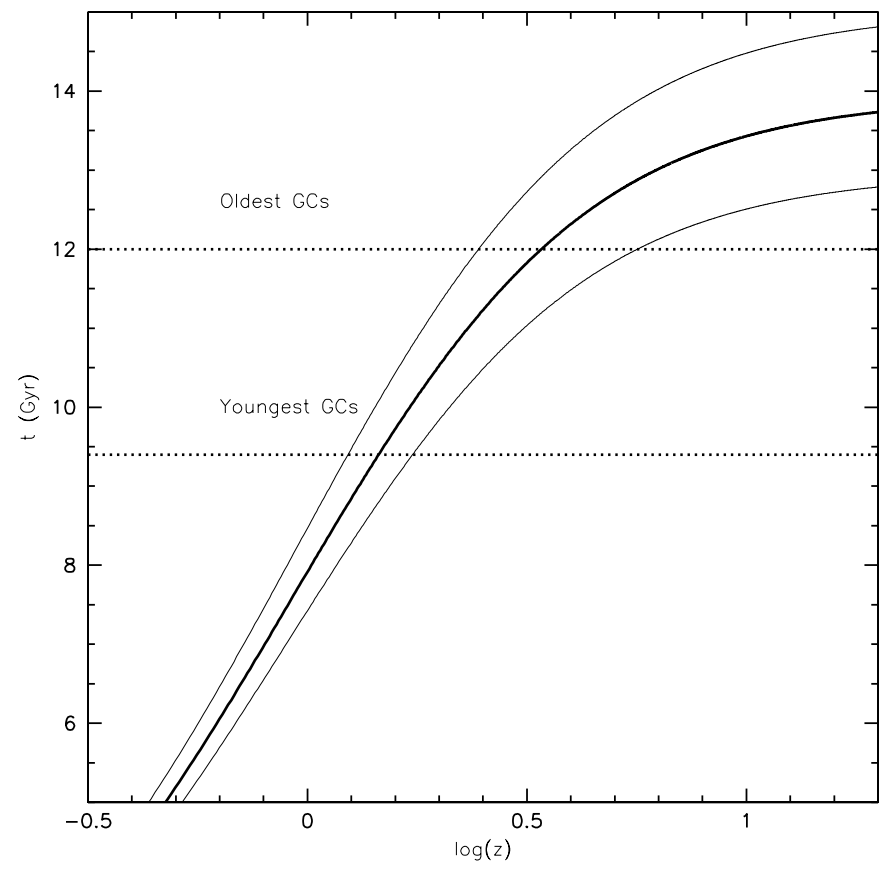

Fig. 5. Age as a function of redshift $z$ for standard $\Lambda$ CDM Universe models. The thick solid line represents the curve obtained for the best choice of parameters given by Spergel et al. (2003); the thin solid lines are obtained summing 1- $\sigma$ variations of the parameters (assumed to be independent to each other). Dashed lines are the 1- $\sigma$ lower limits to the age of the oldest (NGC 6397 and NGC 6752) and youngest globular clusters (47 Tuc).

An alternative use of our age estimate is to constrain the exponent $w$ of the equation of state for the dark energy, as described in Jimenez et al. (2003). An adequate discussion requires the use of cosmological models; this is out of the purposes of this paper. However, we note that our age estimate is more precise and compelling than that considered by Jimenez et al. (2003), so that more stringent lower limits to the time averaged value of $w$ may be derived by combining our age estimate with the location and shape of the first acoustic peak in the microwave background spectrum (Jimenez et al. 2003).

\subsection{The epoch of Galaxy formation}

It is widely assumed that Galactic globular clusters probe the formation of halo and thick disk of our Galaxy (Peebles \& Dicke 1968). This is supported by their kinematics (Zinn 1985), chemical composition (Carney 1993), and colours (Gilmore et al. 1995). However, it should be recalled that in some external galaxies clusters are systematically bluer than the underlying field population, indicating significant systematic differences (see e.g. Harris 1991). On the whole, it seems however reasonable to infer from the age of globular clusters the epoch of formation of the earliest stellar populations in our Galaxy. An earlier attempt in this sense was made by Carretta et al. (2000), who concluded that Galactic globular clusters formed at $z>1$. A much more refined evaluation is possible now, thanks to our new determinations of the ages of NGC 6397, NGC 6752, and 47 Tuc, and the very recent and precise estimates of the age and geometry of the Universe from the WMAP experiment. In a standard $\Lambda$ CDM scenario (Bennett et al. 2003; Spergel et al. 2003) according to WMAP the age of the Universe is $13.7 \pm 0.2 \mathrm{Gyr}$; the Hubble constant (thanks also to data by the HST Key Project: Freedman et al. 2001) is $H_{0}=71 \pm 4 \mathrm{~km} \mathrm{~s}^{-1} \mathrm{Mpc}^{-1}$; and the values of $\Omega_{\mathrm{M}}$ and $\Omega_{\Lambda}$ are fixed at $0.27 \pm 0.04$ and $0.73 \pm 0.04$ respectively.

When coupled with our age estimate for the oldest Galactic globular clusters of $13.4 \pm 0.8 \pm 0.6 \mathrm{Gyr}$, this leads to the conclusion that they formed within 1.7 Gyr from the Big Bang at $1-\sigma$ level of confidence; this corresponds to a redshift of $z>$ 2.5 (see Fig. 5).

Our age for 47 Tuc, as well as the relative age estimates by Rosenberg et al. (1999) and Salaris \& Weiss (2002), indicate that the epoch of formation of globular clusters lasted about 2.6 Gyr, ending about $10.8 \pm 1.4 \mathrm{Gyr}$ ago, that corresponds to a redshift of $z>1.3$. The reason for the absence of a significant population of younger globular clusters in the Galaxy is not entirely understood; however, it is likely to be connected with the presence of a thin disk. In fact, several authors since Peebles \& Dicke (1968) related the large overdensities required to the formation of globular clusters to violent phases connected to accretion episodes, that would have disrupted or at least considerably heated the thin disk. It is then interesting that a phase of low star formation followed the formation of the thick disk (see Gratton et al. 1996, 2000; Fuhrmann 1998; Liu \& Chaboyer 2000), and of its population of globular clusters, like 47 Tuc (Zinn 1985), before the formation of the thin disk started. After this phase, only a few globular clusters actually formed; most of these objects (like e.g. Pal 12) are likely connected to the Sagittarius dwarf galaxy (see e.g. Dinescu et al. 2000).

In the standard $\Lambda C D M$ the end of the phase of formation of globular clusters in our Galaxy corresponds to a redshift of $z>$ 1.3, and suggests a close link between the epoch of formation of the Milky Way spheroid, and that of the early spheroids in high redshift galaxies (Madau et al. 1998).

Acknowledgements. A special thanks goes to P. Montegriffo for making available to us his software. We thank the anonymous referee for his/her useful suggestions that helped to improve the paper. This research has made use of the SIMBAD data base, operated at CDS, Strasbourg, France; it was funded by Ministero Università e Ricerca Scientifica, Italy, through COFIN 2001028897.

\section{References}

Alcaino, G., Liller, W., Alvarado, F., et al. 1997, AJ, 114, 1067

Alonso, A., Arribas, S., \& Martinez-Roger, C. 1996, A\&AS, 117, 227

Bedin, L. R., Piotto, G., Anderson, J., \& King, I. R. 2003, in ASP Conf. Ser., 296, in press [astro-ph/0303291]

Benedict, G. F., McArthur, B. E., Fredrick, L. W., et al. 2002, AJ, 123 , 473

Bennett, C. L., Halpern, M., Hinshaw, G., et al. 2003, ApJ, submitted [astro-ph/0302207]

Cacciari, C., Clementini, G., Castelli, F., \& Melandri, F. 2000, in The Impact of Large Scale Surveys on Pulsating Stars Research, ed. L. Szabados, \& Kurtz (San Francisco: ASP), ASP Conf. , 203, 176

Cacciari, C., \& Clementini, G. 2003, in Stellar Candles (Berlin: Springer), ed. W. Gieren, \& D. Alloin, in press [astro-ph/0301550] 
Caloi, V., D’Antona, F., \& Mazzitelli, I. 1997, A\&A, 320, 823

Caputo, F., Castellani, V., Marconi, M., \& Ripepi, V. 2000, MNRAS, 316,819

Carney, B. W. 1993, in The Globular Cluster-Galaxy Connection, ed. G. H. Smith, \& J. P. Brodie, ASP Conf. Ser., 48, 234

Carney, B. W., Latham, D. W., Laird, J. B., \& Aguilar, L. A. 1994, AJ, 107,2240

Carretta, E., \& Gratton, R. G. 1997, A\&AS, 121, 95

Carretta, E., Gratton, R. G., Clementini, G., \& Fusi Pecci, F. 2000, ApJ, 533, 215

Carretta, E., Gratton, R. G., \& Lucatello, S. 2003, A\&A, submitted

Cassisi, S., Castellani, V., Degl'Innocenti, S., Salaris, M., \& Weiss, A. 1999, A\&AS, 134, 103

Cassisi, S., Salaris, M., \& Irwin, A. W. 2003, ApJ, 588, 862

Castelli, F., Gratton, R. G., \& Kurucz, R. L. 1997, A\&A, 318, 841

Cayrel, R., Hill, V., Beers, T. C., et al. 2001, Nature, 409, 691

Chaboyer, B., Demarque, P., Kernan, P. J., \& Krauss, L. M. 1996, Science, 271, 957

Chaboyer, B., \& Krauss, L. M. 2002, ApJ, 567, L45

Chaboyer, B., Fenton, W. H., Nelan, J. E., Patnaude, D. J., \& Simon, F. E. 2002, ApJ, 562, 521

Clementini, G., Carretta, E., Gratton, R. G., et al. 1995, AJ, 110, 2319

Clementini, G., Carretta, E., Gratton, R. G., \& Sneden, C. 1999, MNRAS, 302, 22

Clementini, G., Gratton, R. G., Bragaglia, A., et al. 2003, AJ, 125, 1309

Cowan, J. J., Sneden, C., Burles, S., et al. 2002, ApJ, 572, 861

Crawford, D. L., \& Mandwewala, N. 1976, PASP, 88, 917

D’Antona, F., Caloi, V., Montalban, J., Ventura, P., \& Gratton, R. 2002, A\&A, 395, 69

Demarque, P., Zinn, R., Lee, Y-W., \& Yi, S. 2000, AJ, 119, 1398

De Marchi, G., Paresce, F., \& Li Causi, G. 2002, BAAS, 201, 712

Dinescu, D. L., Majewski, S. R., Girard, T. M., \& Cudworth, K. M. 2000, AJ, 120, 1892

Dominguez, I., Höflich, P., \& Straniero, O. 2001, ApJ, 557, 279

Fernley, J., Carney, B. W., Skillen, J., Cacciari, C., \& Janes, K. 1998, MNRAS, 293, L61

Ferraro, F. R., Messineo, M., Fusi Pecci, F., et al. 1999, AJ, 118, 1738

Freedman, W. L., Madore, B. F., Gibson, B. K., et al. 2001, ApJ, 553, 47

Freeman, K., \& Bland-Hawthorn, J. 2002, ARA\&A, 40, 487

Fuhrmann, K. 1998, A\&A, 338, 161

Gilmore, G., Wyse, R. F. G., \& Jones, J. B. 1995, AJ, 109, 1095

Girardi, L., Bertelli, G., Bressan, A., et al. 2002, A\&A, 391, 195

Gratton, R. G. 1998, MNRAS, 296, 739

Gratton, R. G., Carretta, E., Matteucci, F., \& Sneden, C. 1996, in Formation of the Galactic Halo... Inside and Out, ed. H. Morrison, \& A. Sarajedini, ASP Conf. Ser., 92, 307

Gratton, R. G., Fusi Pecci, F., Carretta, E., et al. 1997, ApJ, 491, 749

Gratton, R. G., Carretta, E., Matteucci, F., \& Sneden, C. 2000, A\&A, 358,671

Gratton, R. G., Bonifacio, P., Bragaglia, A., et al. 2001, A\&A, 369, 87

Gratton, R. G., Carretta, E., Claudi, R., Lucatello, S., \& Barbieri, M. 2003, A\&A, 404, 187

Grundahl, F., \& Andersen, M. I. 1999, Ap\&SS, 265, 197

Grundahl, F., Stetson, P. B., \& Andersen, M. I. 2002, A\&A, 395, 481

Hansen, B. M. S., Brewer, J., Fahlman, G. G., et al. 2002, ApJ, 574, L155

Harris, W. E. 1991, ARA\&A, 29, 543

Harris, W. E. 1996, AJ, 112, 1487

Hauck, B., \& Mermilliod, M. 1998, A\&AS, 129, 431
Hesser, J. E., Harris, W. E., VandenBerg, D. A., et al. 1987, PASP, 99, 739

Hill, V., Plez, B., Cayrel, R., et al. 2002, A\&A, 387, 560

Houdashelt, M. L., Bell, R. A., \& Sveigart, A. V. 2000, AJ, 119, 1448

Ivans, I. I., Sneden, C., Kraft, R. P., et al. 1999, AJ, 118, 1273

Jimenez, E., Verde, L., Treu, T., \& Stern, D. 2003, ApJ, submitted [astro-ph/0302560]

Kaluzny, J. 1997, A\&AS, 122, 1

King, I., \& Anderson, J. 2002, BAAS, 201, 5509

Koen, C., \& Laney, D. 1998, MNRAS, 301, 582

Kraft, R. P., \& Ivans, I. I. 2003, PASP, 115, 143

Kurucz, R. L. 1994, CD-ROM 13 and CD-ROM 18

Liu, W. M., \& Chaboyer, B. 2000, ApJ, 544, 818

Lutz, T. E., \& Kelker, D. H. 1973, PASP, 85, 573

Madau, P., Pozzetti, L., \& Dickinson, M. 1998, ApJ, 498, 106

Peebles, P. J. E., \& Dicke, R. H. 1968, ApJ, 154, 891

Percival, S., Salaris, M., van Wyk, F., \& Kilkenny, D. 2002, ApJ, 573, 174

Perlmutter, et al. 1999, ApJ, 517, 565

Perryman, M. A. C., Lindegren, L., Kowalewsky, J., et al. 1997, A\&A, 323,49

Pont, F., Mayor, M., Turon, C., \& VandenBerg, D. A. 1998, A\&A, 329,87

Popowski, P., \& Gould, A. 1998, ApJ, 506, 259

Reid, I. N. 1997, AJ, 114, 161

Reid, I. N. 1998, AJ, 115, 204

Renzini, A., \& Fusi Pecci, F. 1988, ARA\&A, 26, 199

Renzini, A., Bragaglia, A., Ferraro, F. R., et al. 1996, ApJ, 465, L23

Rey, S-C., Lee, Y-W., Joo, J-M., Walker, A., \& Baird, S. 2000, AJ, 119,1824

Rich, R. M., Corsi, C. E., Bellazzini, M., et al. 2002, in Extragalactic Star Clusters, ed. E. K. Grebel, D. Geisler, \& D. Minniti (Astronomical Society of the Pacific), IAU Symp., 207, 140

Richard, O., Michaud, G., Richer, J., et al. 2002, ApJ, 568, 979

Richer, H. B., Hansen, B., Limongi, M., et al. 2000, ApJ, 529, 318

Rosati, P., Borgiani, S., \& Norman, C. 2002, ARA\&A, 40, 539

Rosenberg, A., Saviane, I., Piotto, G., \& Aparicio, A. 1999, AJ, 118, 2306

Salaris, M., \& Weiss, A. 2002, A\&A, 388, 492

Sandage, A. 1990, ApJ, 350, 603

Sandage, A. 1993, AJ, 106, 719

Schlegel, D. J., Finkbeiner, D. P., \& Davis, M. 1998, ApJ, 500, 525

Schuster, W. J., \& Nissen, P. E. 1989, A\&A, 221, 65

Sneden, C., Cowan, J. J., Ivans, I., et al. 2001, ApJ, 533, L139

Spergel, D. N., Verde, L., Peiris, H. V., et al. 2003, ApJ, submitted [astro-ph/0302209]

Straniero, O., Chieffi, A., \& Limongi, M. 1997, ApJ, 490, 425

Storm, J., Carney, B. W., \& Latham, D. W. 1994, A\&A, 290, 443

Thompson, I. B., Kaluzny, J., Pych, W., \& Krzeminski, W. 1999, AJ, 118,462

Tsujimoto, T., Miyamoto, M., \& Yoshii, Y. 1998, ApJ, 492, L79

Truran, J. W., Cowan, J. J., Pilachowski, C. A., \& Sneden, C. 2002, PASP, 114, 1293

VandenBerg, D. A., Swenson, F. J., Rogers, F. J., Iglesias, C. A., \& Alexander, D. R. 2000, ApJ, 532, 430

VandenBerg, D. A., Richard, O., Michaud, G., \& Richer, J. 2002, ApJ, 571,487

Wang, et al. 2000, ApJ, 530, 17

Zinn, R. 1985, ApJ, 293, 424

Zoccali, M., Renzini, A., Ortolani, S., et al. 2001, ApJ, 553, 733 